\title{
Self-medication with antibiotics among seafarers: a public health issue
}

\author{
Yusuff Adebayo Adebisi $^{1}{ }^{\oplus}$, lyiola Olatunji Oladunjoye ${ }^{2}{ }^{\oplus}$, \\ Yusuf Amuda Tajudeen ${ }^{2}$, Don Eliseo Lucero-Prisno III $^{3}$ \\ ${ }^{1}$ Faculty of Pharmacy, University of Ibadan, Nigeria \\ ${ }^{2}$ Department of Microbiology, Faculty of Life Sciences, University of Ilorin, Nigeria \\ ${ }^{3}$ Department of Global Health and Development, London School of Hygiene and Tropical Medicine, United Kingdom
}

Self-medication is the use of drugs by individuals for self-diagnosed illnesses or symptoms without proper diagnosis and therapeutic interventions by qualified health professionals. A major driver of antibiotic resistance in humans is the act of self-medication which leads to the overuse, underuse, and misuse of antibiotics [1]. With the emergence of the coronavirus disease 2019 (COVID-19) pandemic, the use of antibiotics has gained rapid usage, further complicating the fight against antimicrobial resistance [1]. Therefore, it is essential that we continue to monitor antibiotics usage and strengthen surveillance in different communities, including among seafarers. To the best of our knowledge, and searches conducted across major scientific databases (Medline, Scopus, PubMed, Google Scholar, and PubMed Central), there is a major dearth of studies or reported data on self-medication with antibiotics among seafarers globally.

There is a possibility of significant self-medication practices with medicines, including antibiotics among seafarers since medications are allowed on board. Additionally, limited knowledge of antibiotics use and lack of awareness of the possible dangers of its misuse can also fuel pervasive misuse of antibiotics for all kinds of illnesses, including viral diseases, among seafarers. In the International Labour Organization/International Maritime Organization Guidelines on Seafarer Medical Examinations, seafarers that require the use of prescription and over-the-counter drugs are advised to always inform the master of any medications brought on board, to avoid a violation of drug and alcohol policy [2]. However, the guideline does not cover monitoring of the usage of these drugs and strategies towards preventing needless antibiotic usage on the sea [2]. This is concerning because alcohol and drug abuse have been reported among seafarers [3]. Furthermore, antibiotic-resistant pathogens of public health implications in humans have been discovered on the sea [4] and in sea animals [5]. This implies there is an urgent need to build research capacity on issues pertaining to antibiotic misuse among seafarers and to formulate measures to curb the misuse and promote antimicrobial stewardship.

We strongly recommend that an awareness campaign is carried out among seafarers to understand the implication of misuse and overuse of antimicrobials and urge strong monitoring on the usage of antimicrobials. We also recommend that maritime scientists perform research on the prevalence of self-medication among seafarers as there are no reports on this and encourage relevant stakeholders to provide adequate support and funding. The editors of the "International Maritime Health" journal should also call for articles aimed at this research.

In conclusion, the International Maritime Organization should collaborate with public health institutions to ascertain the risk of antibiotic usage among seafarers and also understand the extent to which it is occurring to as to set up measures that can curb this public health issue, antibiotic resistance.

\section{Conflict of interest: None declared}

\section{REFERENCES}

1. Adebisi YA, Jimoh ND, Ogunkola IO, et al. The use of antibiotics in COVID-19 management: a rapid review of national treatment guidelines in 10 African countries. Trop Med Health. 2021; 49(1): 51, doi: 10.1186/s41182-021-00344-w, indexed in Pubmed: 34162445.

2. Guidelines on the medical examinations of seafarers. International Labour Organization and International Maritime Organization. 2013. https://www.ilo.org/wcmsp5/groups/public/--ed_dialogu- 
e/--sector/documents/normativeinstrument/wcms_174794.pdf (August 12, 2021)

3. Stoll E, Püschel K, Harth V, et al. Prevalence of alcohol consumption among seafarers and fishermen. Int Marit Health. 2020; 71(4): 265 -274, doi: 10.5603/IMH.2020.0045, indexed in Pubmed: 33394491.

4. Li W, Su H, Cao Y, et al. Antibiotic resistance genes and bacterial community dynamics in the seawater environment of Dapeng
Cove, South China. Sci Total Environ. 2020; 723: 138027 , doi: 10.1016/j.scitotenv.2020.138027, indexed in Pubmed: 32224396.

5. Blasi MF, Migliore L, Mattei D, et al. Antibiotic resistance of gram-negative bacteria from wild captured loggerhead sea turtles. Antibiotics (Basel). 2020; 9(4), doi: 10.3390/antibiotics9040162, indexed in Pubmed: 32268481. 